\title{
ENERGY EFFICIENCY ANALYSIS FOR LTE-A HETEROGENEOUS CELLULAR NETWORK
}

\author{
Diwakar A. Danecha, Kirit G. Bhuva and Sarosh K. Dastoor \\ Department of Electronics and Communication Engineering, Dr. S and S.S. Ghandhy Government Engineering College, India
}

\begin{abstract}
Existing cellular network gives us the large network coverage as well as network capacity but in many cases it fails to achieve predicted data rates for the seamless wireless communication. Moreover there is a tremendous increase in wireless device users so the data rates and network capacity offered by conventional cellular network is not sufficient. So the effort to suppress base-stations power consumption are desperately needed and for that heterogeneous cellular network has been introduced. This paper provides the complete analysis of energy efficient wireless systems for heterogeneous cellular network as it has been pointed out to be one of the key network architectures that help to increase system capacity and reduce power consumption. Heterogeneous network with coordinated multi-point has received significant attention as a way of achieving energy efficiency and to improve the network handling capacity in heterogeneous cellular network. Usually in communication those users which are on the edge of the cells always suffer with the low data rates and low capacity. Moreover they get interference from the adjacent cells. To mitigate these problems and achieve higher energy efficiency Heterogeneous network with coordinated multi-point came out as one of the best solutions. In this paper heterogeneous network is considered with the Voronoi tessellation and between the cells coordinated multi-point technique is applied and compared with the non-coordinated multipoint scenario. Heterogeneous network is the integrated part of the beyond $4 G$ wireless network for better energy efficiency.
\end{abstract}

\section{Keywords:}

Heterogeneous Network, Energy Efficiency, Coordinated Multi-Point, LTE-A, Voronoi Tessellation

\section{INTRODUCTION}

There are a number of reasons why different mobile net- work operators and mobile network device manufacturers are together globally researching different ways to optimize their network performance from the energy efficiency and capacity standpoint [1]. In recent years, with the explosive growth of mobile communications in terms of number of connected devices and the demand for new services and ubiquitous connectivity, the energy consumption of wireless access networks is experiencing a significant increase. Soaring cost of energy is not only problem for mobile operators but these are also big challenges for them.

Deployment of increasingly powerful mobile network technologies has taken place within the last decade. Although network efficiency has been growing, the higher access rates have inevitably led to increased energy consumption in base stations (BSs) and network densities have been constantly growing [2]. Recently, the mobile communication community has become aware of the large and ever-growing energy usage of mobile networks. Yet, the work on the reduction of energy consumption has been mostly carried out by BS manufacturers since high power efficiency provides a competitive advantage [3]. Today one of the most discussed topics is the global warming, which is due to increment of $\mathrm{CO} 2$ (Carbon dioxide) and other greenhouse gases concentration levels in atmosphere [4,5].

Mobile networks do not have considerable share in the overall energy consumption of the ICT sector, which itself is responsible for $2 \%$ to $10 \%$ of the world energy consumption. The reports show that ICT, the fifth largest industry in power consumption, emits $2 \%$ of the world-wide $\mathrm{CO} 2$ representing approximately one fourth of the emissions produced by cars. Despite the fact that these numbers look rather small, they are expected to increase by nearly a factor of 3 due to upward trend in energy consumption. This issue motivates governments to take political action in order to prevent global warming. In 2008, the European Commissions (EC) decided to lower CO2 emissions by $20 \%$ by 2020 [6]. However, reduction in energy consumption of mobile networks is of great importance from economical (cost reduction) and environmental (decreased $\mathrm{CO} 2$ emissions) perspective [7].

Furthermore, energy savings depends significantly on human behavior and utilization of resulting financial savings, as any energy saving is followed by cost savings. From a network operator or infrastructure owner's business perspective, the cost saving factor in many cases is of more importance than environmental protection. Money saved by reducing energy consumption is available for other expenditures. If the saved cost would be invested in a proper way, the low carbon society will be created. Alternatively energy savings could be affected by rebound effect in which the improper utilization of the saved cost might give unfortunate results of potentially additional environmental issues [8].

A typical mobile network consists of three main elements: core network, base stations, and mobile terminals. Base stations contribute $60 \%$ to $80 \%$ of the whole network energy consumption [9]. Thus the efforts in the reduction of energy consumption focus on the BS equipment, which includes the minimization of BS energy consumption, minimization of BS density (BS density is inversely proportion to cell area) and the use of renewable energy sources.

The growing demand for ubiquitous connectivity and new services, however, comes with an undesired consequence of increasing energy consumption. Currently, information and communications technology (ICT) is responsible for $3 \%$ of worldwide electricity consumption out of which wireless access network contributes approximately $10 \%$ at 60 billion $\mathrm{kWh}$ per year [10-12]. This consumption corresponds to typical annual electricity consumption of 20 million European households. Annual electricity consumption has risen 16-20\% every year, corresponding to a twofold increase in every 4-5 years [13].This situation impose a challenge for mobile operators since rising energy consumption together with energy prices directly result in an increase in their OPerational EXpenditures (OPEX). In fact, 
operators cost figures show that nowadays the energy cost of running a network constitutes almost $50 \%$ of overall OPEX. It should be noted that this cost is not only due to the direct cost of electricity, but also comes from the operation of off- (electrical) grid base stations (BSs) in the network. In such cases, especially in many emerging markets, delivering fuel to the BS sites accounts for a significant share of the operator's total energy cost [14].

Coordinate multipoint technique which is introduced in LTEA has come out as a best solution to decreases the energy consumption and to increase the capacity at cell edge users [15]. Improvement of network performance in term of interference management, network capacity and cell edge spectral efficiency is possible by CoMP techniques. In CoMP technique BSs are coordinated with each other and enable multiple transmissions. Due to this user demands are satisfied by providing best services. Dynamic point selection (DPS), joint transmission (JT), and coordinated scheduling/coordinated beamforming (CS/CB) are the three major downlink CoMP techniques introduced by $3 \mathrm{GPP}$ [16], In JT technique, multiple coordinating BSs transmit data simultaneously to a UE and improves the received signal quality. In DPS technique, though UE data is available to multiple coordinating BSs, only one of the BSs is selected for transmission. However, based on the wireless resource availability and channel state information, transmitting BS serving a specific UE can be switched among the coordinating BSs at the sub frame level [16]. On the other hand, in CS/CB technique, UE scheduling/ beamforming decisions are made through coordination among the cooperating BSs, while data for transmission to UE is only available at and transmitted from one BS. Selection of transmitting BS is chosen in a semi-static manner configured by higher-layer radio resource control signaling [16].

Rest of the paper is organized as follows: Section 2 consists the system model which contains network layout brief about used CoMP technique, link model and BSs power consumption model. Section 3 consists simulation setup and analysis of results and section 4 consists conclusion of the carried analysis.

\section{SYSTEM MODEL}

\subsection{NETWORK LAYOUT}

We consider a two-tier heterogeneous LTE-A cellular network having a mix of high-power macro-cells and low-power small cells. In the real scenario we can't consider the shape of cells exactly as hexagonal or circular. So, here voronoi region is considered which can relate with the real scenario of the shape of the cells. A section of the network with a multiple macrocells and several small cells are shown in Fig.1. Random pattern spatial distributions of user equipment's (UEs) inside a region is considered.

In random pattern, as shown in Fig.1, throughout the voronoi coverage region user equipment's are uniformly distributed. This voronoi region with random pattern UEs distribution nearly resembles the real-life random BS locations and coverage areas, there is no restriction for putting two BSs at minimum distance from each other. i.e., two BSs can be very close to one another. However, in practice, BSs must have some geographic distance from neighboring BSs. For emulating such practical phenomena.

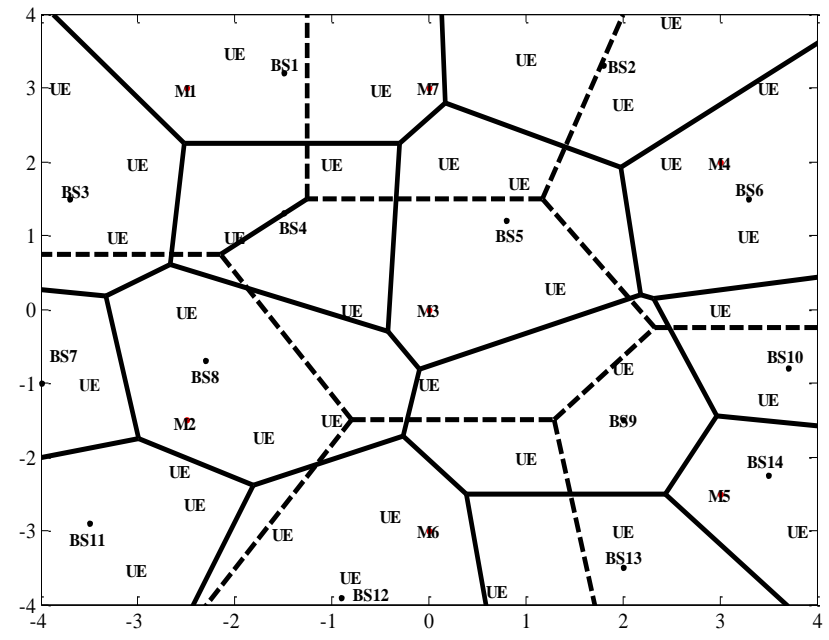

Fig.1. Two-tier heterogeneous network model as a Voronoi tessellation

\subsection{CoMP TECHNIQUES}

CoMP techniques use multi-cell cooperation to mitigate intercell interference and to enhance the desired signal quality of celledge users, thereby primarily improving the cell-edge throughput. There are three types of CoMP techniques. One is Coordinated Scheduling and Beamforming (CS/CB). Second is Dynamic Point Selection (DPS) and third is Joint Transmission (JT). This paper analysis one of the CoMP technique which is Dynamic Point Selection (DPS). In Dynamic Point Selection (DPS), at multiple base stations within the coordinating set the UE data is available but data is only transmitted from one base station at a time. DPS is a simple but effective CoMP scheme that switches the serving a Transmission Point (TP) of the UE based on the UEs channel and the cell loading conditions. This TP switching can be done in a very fast time scale, even on a sub frame to sub frame basis without requiring an elaborate handover procedure. It provides both macro diversity and fast load balancing gains. Macro diversity benefit is obtained by choosing the best serving TP according to the UEs current channel conditions and the fast load balancing gains are realized by transmitting to the UE from the less-loaded TP.

\subsection{LINK MODEL}

We have considered log-normally distributed shadow fading for channel model. So, the received power $P_{i}^{\gamma}$ at $u^{\text {th }}$ UE located at a distance of $d_{i, u}$ from $i^{\text {th }}$ BS can be given by [4],

$$
P_{i}^{\gamma}=P_{i, u}^{t} d_{i, u}^{-\alpha} 10^{\zeta / 10}
$$

where, $P_{i, u}^{t}$ is the transmitted power at $u^{\text {th }} \mathrm{UE}$ from $i^{\text {th }} \mathrm{BS}, \zeta$ is a Gaussian random variable with mean zero and standard deviation $\sigma \mathrm{dB}$, and $\alpha$ is the path-loss exponent. Then, the received SINR $\gamma_{i, u}$ at $u^{\text {th }} \mathrm{UE}$ from $i^{\text {th }}$ BS can be given by,

$$
\gamma_{i, u}=\frac{P_{i, u}^{\gamma}}{I_{u, i n t e r}+I_{u, i n t r a}+P_{N}}
$$

where, $I_{u, \text { inter }}$ is the inter-cell interference, $I_{u \text {,intra }}$ is the intra-cell interference, $P_{N}$ is the additive white Gaussian noise (AWGN) power given by,

$$
P_{N}=-174+10 \log _{10}(\Delta f)
$$


where, $P_{N}$ is measured in $\mathrm{dBm}$ with $\Delta f$ is the bandwidth in $\mathrm{Hz}$.

\subsection{BSs POWER CONSUMPTION MODEL}

We have considered that both macro and small cells are operated in active mode as well as passive mode according to the serving availability of the UE. Which means if there is no UE to be served than cell will be operated in the sleep mode otherwise in active mode. In DSP only one BS is transmitting at a time so other is considered in sleep mode and the BS which is transmitting is considered as in active mode.

Table.1. BS Power Consumption Model Parameters [12]

\begin{tabular}{|c|c|c|c|c|c|}
\hline BS Type & $\mathbf{N}_{\text {Trx }}[\mathbf{W}]$ & $\mathbf{P}_{\text {Max }}[\mathbf{W}]$ & $\mathbf{P}_{\mathbf{0}}[\mathbf{W}]$ & $\boldsymbol{\Delta} \mathbf{P}$ & $\mathbf{P}_{\text {s }}[\mathbf{W}]$ \\
\hline Macro & 1 & 20.0 & 130.0 & 4.7 & 75.0 \\
\hline Small & 1 & 0.13 & 6.8 & 4 & 4.3 \\
\hline
\end{tabular}

We have considered liner power consumption model so total power consumption of the base station can be given by,

For active mode,

$$
P_{C}=N_{T R X} P_{O}+P_{\text {OUT }} \Delta P
$$

For sleep mode,

$$
P_{C}=N_{\text {TRX }} P_{\text {sleep }}
$$

where, NTRX is the number of transceivers per BS, $P_{M A X}$ is the maximum transmit power of an active $\mathrm{BS}, 0 \leq P_{\text {OUT }} \leq P_{\text {MAX }}$ is the actual transmit power, $P_{\text {sleep }}$ is the sleep mode power consumption, $P_{0}$ is the power consumption at zero output power when no UE is served and $\Delta P$ is the slope of the load-dependent power consumption profile.

\subsection{PERFORMANCE METRICS}

Throughput of the given network can be calculated by the Shannon's capacity formula which is given below,

$$
R_{\text {total }}=\sum_{u=1}^{U} \sum_{i=1}^{N_{a}, u+1} \Delta f \log _{2}\left(1+\gamma_{i, u}\right)
$$

where, NS, $U$ is the number of transmitting small cells for serving $\mathrm{UE}$ and $U$ is the total number of UEs in the network.

Total power consumed by the network is given by,

$$
P_{\text {total }}=\sum_{m=1}^{M_{\text {active }}} P_{m, \text { active }}+\sum_{m=1}^{M_{\text {sleep }}} P_{m, \text { sleep }}
$$

where, $M_{\text {sleep }}$ and $M_{\text {active }}$ are total number of the active and sleep mode BSs respectively. $P_{m}$, active and $P_{n}$, sleep are total power consumption in $m^{\text {th }}$ active mode and $n^{\text {th }}$ sleep mode BSs respectively.

Evaluation the EE performance of the network with the proposed CoMP technique in terms of bits per joule, which is defined as the ratio of aggregate throughput $R_{\text {total }}$ of the network to the total power consumed by the network $P_{\text {total }}$. Thus the EE metric denoted as $\eta_{E E}$ can be written as,

$$
\eta_{E E}=\frac{R_{\text {total }}}{P_{\text {total }}}
$$

Then, the normalized $\eta_{E E}$ of the proposed network can be evaluated by taking the ratio of EE metric found in Eq.(8) to that of the traditional non-CoMP based cellular networks with macrocell only. This unit less normalized $\eta_{E E}$ implies the EE improvement by the proposed CoMP technique over the conventional macrocell non-CoMP systems.

\section{RESULTS AND ANALYSIS}

\subsection{SIMULATION SETUP}

Performance of the CoMP technique in term of SINR and EE is evaluated through matlab simulation. Voronoi tessellation is considered as shown in the Fig.1. Network is considered 2-tier as shown in Fig.1. 2-tiers can be differentiated by the blue and red colors. Macro cells stations are denoted as $M_{1}, M_{2}, M_{3}, \ldots, M_{7}$. Small cells stations are denoted as $\mathrm{BS}_{1}, \mathrm{BS}_{2}, \mathrm{BS}_{3}, \ldots, \mathrm{BS}_{14}$. Into the region randomly UEs are distributed.

All the BSs are considered as omnidirectional antennas with carrier frequency of $2 \mathrm{GHz}$ and channel bandwidth $5 \mathrm{MHz}$ and $1.4 \mathrm{MHz}$ are considered for macrocell and small cell respectively. For the channel, a path-loss exponent of 3.574 and shadow fading standard deviation $8 \mathrm{~dB}$ is used for the simulations [17].

Table.2. Simulation Parameter

\begin{tabular}{|c|c|}
\hline Parameters & Values \\
\hline Carrier Frequency $f$ & $2 \mathrm{GHz}$ \\
\hline Channel Bandwidth $\Delta f$ & $1.4 \mathrm{MHz}$ \\
\hline Total Resource Blocks $(\mathrm{RBs})$ & 6 \\
\hline Transmitted Power $P_{t}$ & $43 \mathrm{dBm}$ \\
\hline Path-loss Exponent $\alpha$ & 3.574 \\
\hline Gaussian Random variable $\xi$ & 2 \\
\hline Shadowing Standard Deviation $\sigma$ & $8 \mathrm{~dB}$ \\
\hline
\end{tabular}

The Fig. 2 represents the cumulative distribution function (CDF) plot of the EE for the both CoMP and Non-CoMP scenarios. In this Fig.2 it is seen that energy efficiency is higher and tends to reach 1 in CoMP scenario in compare with Non-CoMP scenario. Non-CoMP has poor performance as its EE is lower than CoMP.

The Fig. 3 represents the CDF plot of the throughput for both CoMP and Non-CoMP scenarios. From Fig.3, it is seen that CoMP provides higher throughput in compare with Non-CoMP. Main goal of LTE-A is to achieve higher system capacity as well as datarates. By using this CoMP techniques, LTE-A goals can be satisfied.

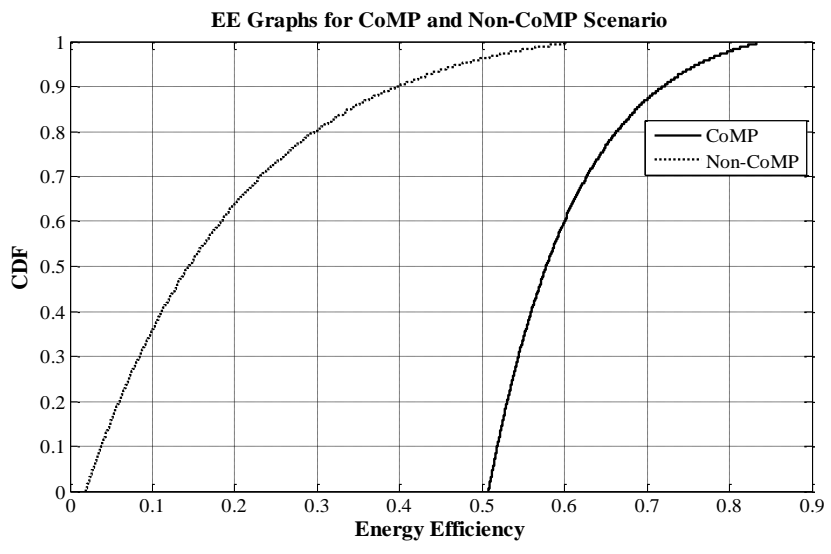

Fig.2. CDF Plot of EE 


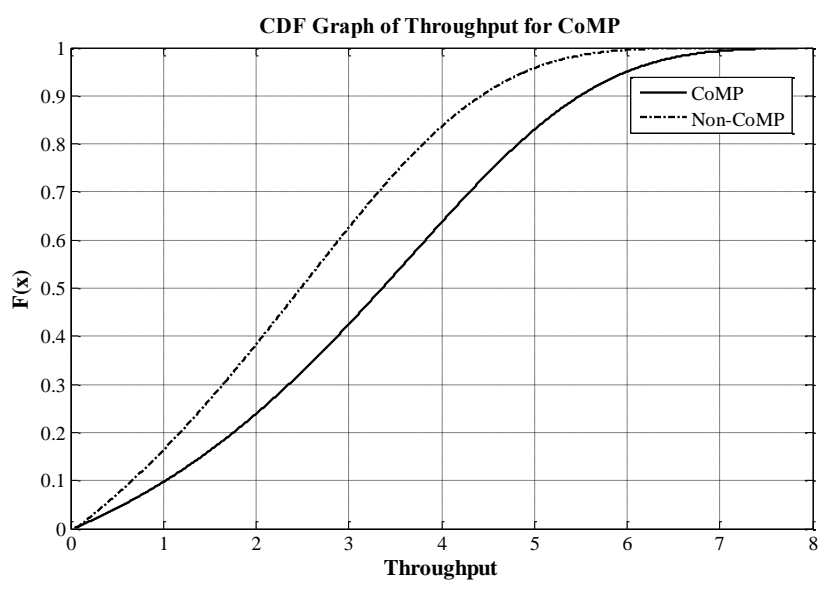

Fig.3. CDF Plot of Throughput

The Fig.4 shows the distance vs throughput graph. CoMP is nothing but the Coordinate multipoint which means BSs are coordinated and provide smooth performance for users at cell edge. Due to this coordination the coverage of the cells also increases and this increased coverage is one of the properties of CoMP. From Fig.4 it is seen that the distance covered by CoMP is approx. $18 \mathrm{kms}$ whereas distance covered by Non-CoMP is $15 \mathrm{~km}$. So by using CoMP $3 \mathrm{~km}$ extension can be achieved.

The Fig.5 represents SINR vs. BER plot. It is seen than in CoMP the bit error rate (BER) is constantly decrease as SINR increases. While in Non-CoMP, it is almost constant at higher value in compare with CoMP. As BER values are vary lower in CoMP scenario so it gives better performance than Non-CoMP.

The explained DPS CoMP technique is the best in terms of $\mathrm{EE}$, which gives higher performance.

\section{CONCLUSION}

This paper has investigated DPS CoMP technique for the twotier heterogeneous LTE-A cellular networks and thoroughly investigated its EE performance. Voronoi tessellation with macro cells and small cells with randomly distributed UEs have been considered. Through simulation energy efficiency performance has been investigated by using DPS CoMP for heterogeneous cellular network. EE performance of the proposed CoMP technique has also been clearly identified. This paper concludes that energy efficiency reaches its optimum value earlier by using DPS CoMP in compare with Non-CoMP. Comparison of the DPS CoMP technique with that of Non-CoMP transmission techniques have also shown its superior EE performance for wide range of network scenarios. Thus, coordinated multi-point techniques have the great potential to improve the performance of the cellular network. Future scope in this paper will be to analyze that how frame by frame the transmission point is changing when DPS is used. Also, to impose the Voronoi tessellation on any particular earth region and to analyze same for real time scenario.

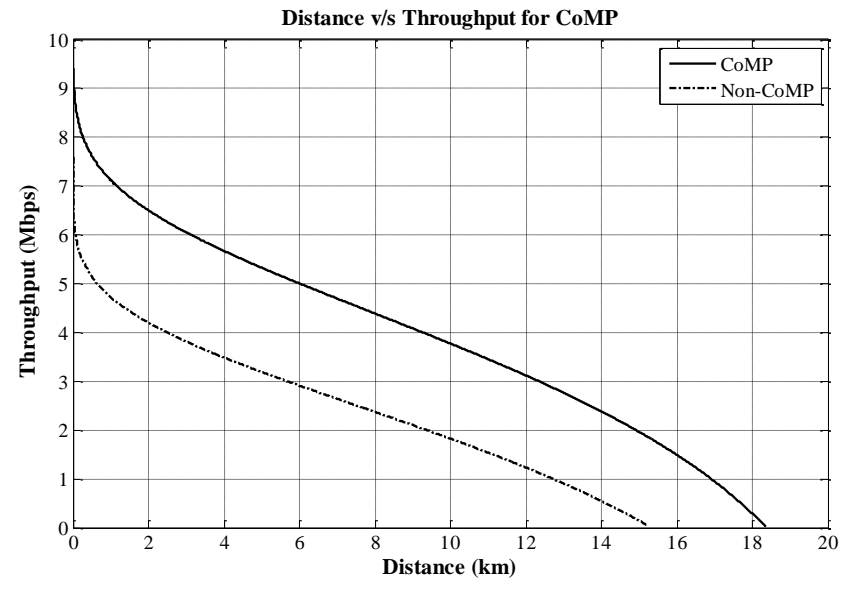

Fig.4. Distance vs Throughput

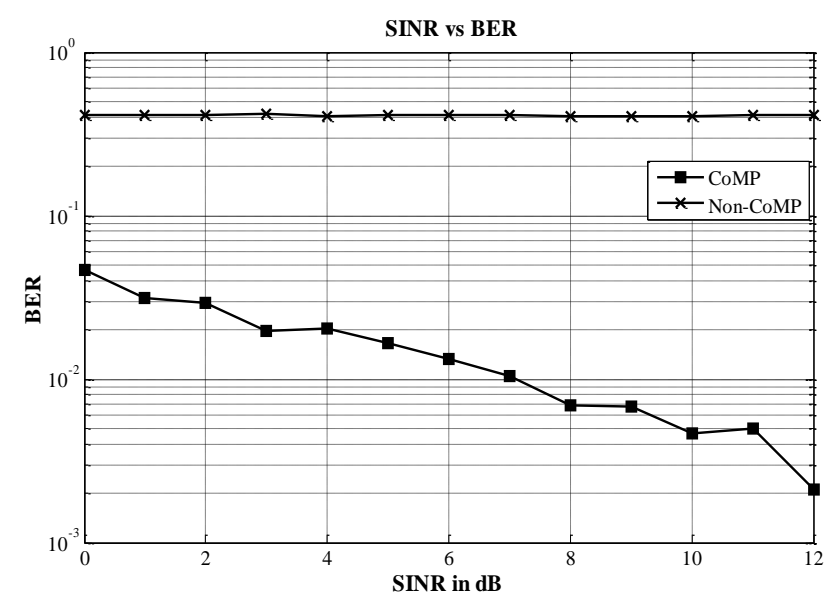

Fig.5. SINR vs BER

\section{REFERENCES}

[1] R. Samuelson, "The Moral Challenge of Globalization", Washington Post, 2008.

[2] Steve Roy and Global Marketing, "Energy Logic for Telecommunications", White Paper, Emerson Network Power, pp. 1-15, 2008.

[3] Sustainable Energy use in Mobile Communications, Available at: https://www.techonline.com/electricalengineers/education-training/tech-

papers/4136182/Sustainable-Energy-Use-in-MobileCommunications.

[4] $\mathrm{CO}_{2}$ the Major Cause of Global Warming, Available at: http://timeforchange.org/CO2-cause-of-global-warming.

[5] Earth Project, Available at: http://www.accessnets.org/keynote.shtml.

[6] C. Bohringer, T.F. Rutherford and R.S. Tol, "The EU 20/20/2020 Targets: An Overview of the EMF22 Assessment", Energy Economics, Vol. 31, pp. 268-273, 2009.

[7] M. De Sanctis, E. Cianca and V. Joshi, "Energy Efficient Wireless Networks towards Green Communications", Wireless Personal Communications, Vol. 59, No. 3, pp. 537552, 2011. 
[8] H.O. Scheck, "ICT and Wireless Networks and their Impact on Global Warming", Proceedings of European IEEE Wireless Conference, pp. 911-915, 2010.

[9] J.T. Louhi, "Energy Efficiency of Modern Cellular Base Stations", Proceedings of $29^{\text {th }}$ IEEE International Telecommunications Energy Conference, pp. 475-476, 2007.

[10] A. Fehske, G. Fettweis, J. Malmodin and G. Biczok, "The Global Footprint of Mobile Communications: The Ecological and Economic Perspective", IEEE Communications Magazine, Vol. 49, No. 8, pp. 55-62, 2011.

[11] S. Tombaz, A. Vstberg and J. Zander, "Energy and Cost Efficient Ultra High Capacity Wireless Access", IEEE Wireless Communications Magazine, Vol. 18, No. 5, pp. 1824, 2011.

[12] G. Auer et al., "How Much Energy is Needed to Run a Wireless Network?", IEEE Wireless Communications Magazine, Vol. 18, No. 5, pp. 40-49, 2011.

[13] Gerhard Fettweis and Ernesto Zimmermann, "ICT Energy Consumption: Trends and Challenges", Proceedings of Wireless Personal Multimedia Communications, pp. 1-4, 2008.

[14] S.T. Ericsson, "Enhanced Network Energy Efficiency (R1100062)", TSG-RAN Working Group Meeting, 2010.

[15] Ian F. Akyildiz, David M. Gutierrez-Estevez and Elias Chavarria Reyes, "The Evolution to 4G Cellular Systems:
LTE-Advanced”, Physical Communication, Vol. 3, No. 4, pp. 217-244, 2010.

[16] J. Lee et al., "Coordinated Multipoint Transmission and Reception in LTE-Advanced Systems", IEEE Communications Magazine, Vol. 50, No. 11, pp. 44-50, 2012.

[17] Juha Meinila et al., "D5. 3: Winner+ Final Channel Models", Available at: http://projects.celticinitiative.org/winner+/WINNER+\%20Deliverables/D5.3_v 1.0.pdf.

[18] Kazi Mohammed Saidul Huq et al., "Green HetNet CoMP: Energy Efficiency Analysis and Optimization", IEEE Transactions on Vehicular Technology, Vol. 64, No. 10, pp. 4670-4683, 2015.

[19] K. Son, E. Oh and B. Krishnamachari, "Energy-Efficient Design of Heterogeneous Cellular Networks from Deployment to Operation", Computer Networks, Vol. 78, pp. 95-106, 2015.

[20] C.C. Hsu and J. Chang, "Spectrum-Energy Efficiency Optimization for Downlink LTE-A for Heterogeneous Networks", IEEE Transactions on Mobile Computing, Vol. 16, No. 5, pp. 1449-1461, 2017.

[21] S. Boiardi, A. Capone and B. Sans, "Radio Planning of Energy-Aware Cellular Networks", Computer Networks, Vol. 57, No. 13, pp. 2564-2577, 2013. 